\title{
Effect of Micro-Mixing on the Yield of Intermediates in Triplet Consecutive/Competitive Reactions
}

\author{
AMER M. EL-HAMOUZ ${ }^{1}$ and REGINALD MANN ${ }^{2 *}$ \\ ${ }^{1}$ Department of Chemical Engineering, An-Najah National University, Nablus, West Bank, via Israel \\ ${ }^{2}$ Department of Chemical Engineering, UMIST, Manchester, M60 1QD, England
}

\begin{abstract}
Micro-mixing calculations based upon a simplified non-deforming one-dimensional diffusion-reaction formulation are presented for the sequence of three reactions denoted by $A+B \rightarrow R+B \rightarrow S+B \rightarrow T$. This triplet reaction under micro-mixing is posed as five simultaneous parabolic partial differential equations which have been solved using a general purpose NAG library algorithm. The primary product $R$ has an improved yield under perfect mixing conditions. The ultimate product $T$ is shown to be always favoured by the perfect segregation of micro-mixing. By contrast, the central intermediate $\mathrm{S}$ gives high yields under micro-mixing at low conversions, but perfect mixing is preferable at higher conversions. In addition, predictions show that yield improvements for $S$ might be possible by an engineered stretching of fluid lamellae and by the combination of micro-mixing followed by perfect mixing, especially at intermediate levels of conversion. The calculations are relevant to multi-step halogenations and they help to explain how the anomolous appearance of a tri-halogenated species ahead of mono- and di-halogenated ones in a stirred vessel can be attributed to micro-mixing.
\end{abstract}

Des calculs de micromélange s'appuyant sur une formulation de diffusion-réaction unidimensionnelle non déformante simplifiée sont présentés pour une séquence de trois réactions notées par $A+B \rightarrow R+B \rightarrow S+B \rightarrow T$. En micromélange, ce triplet s'exprime sous la forme de cinq équations différentielles partielles paraboliques simultanées qui ont été résolues au moyen d'un algorithme général de la bibliothèque NAG. Le produit primaire $\mathrm{R}$ a un rendement amélioré dans des conditions de mélange parfaites. On montre que le produit final $T$ est toujours favorisé par la ségrégation parfaite du micromélange. À l'opposé, le rendement en produit intermédiaire S est très élevé pour un micromélanage à faible conversion, mais un mélange parfait est préférable à une conversion élevée. De plus, les prédictions montrent que des améliorations du rendement en $S$ pourraient être possibles par un étirement des lamelles de fluide et par la combinaison d'un micromélange suivi d'un mélange parfait, particulièrement aux niveaux intermédiaires de conversion. Ces calculs sont pertinents pour les halogénations multiétagées et aident à expliquer comment l'apparence anormale d'une espèce trihalogénée devant des espèces mono et dihalogénées dans un réservoir agité peut être attribuée au micromélange.

Keywords: micro-mixing, consecutive/competitive reactions, chemical selectivity, chemical yield.

$\mathrm{I}_{\mathrm{n}}^{\mathrm{n}}$ the field of chemical reaction engineering, it is probably true to say that the process of achieving a molecular scale of mixing, so-called micro-mixing, is not yet perfectly well understood, especially for cases with multiple reactions with non-linear kinetics. Furthermore, analysis of micro-mixing problems still encounters difficulties with intensive computation as well as conceptual problems matching mathematical models to physical realities of fluids at the micro-scale. Micro-mixing is the most indispensible mixing process for promoting reactions since, without it, no reactions could proceed.

The basic concepts of segregation intensity (Danckwerts, 1958) and the extremes of maximum mixedness and complete segregation (Zweitering, 1959) have been slow to develop and practical applications of these fundamental ideas have been relatively few over the 40 years since they were first introduced.

Applications to reactors initially relied upon two kinds of models. The coalescence/redispersion models (Spielman and Levenspiel, 1965; Treleaven and Tobgy, 1972) apply the notion of the interaction of myriad separate packets of segregated fluid. The multi-environment models envisage distinct environments, which are perfectly micro-mixed or perfectly segregated, which interact with one another (Weinstein and Adler, 1967; Ritchie and Tobgy, 1979; Mehta and Tarbell, 1983). Subsequent developments tended to exploit the best features of these two approaches and the

\footnotetext{
*Author to whom correspondence should be addressed. E-mail address: r.mann@umist.ac.uk
}

"interaction and exchange with mean model (IEM)" was shown to be a practical proposition for direct application to typical stirred reactors (Villermaux et al., 1989).

Examples of reactor calculations for a triplet sequence of competitive/consecutive reactions have usually ignored the question of micro-mixing (McMullin, 1948; Haring and Knol, 1964; Russell and Buzzelli, 1969) and have assumed perfect mixing. The results of Haring and Knol for the sidechain repeated substitution of toluene by chlorine, exhibit the classical sequential appearance of the mono-, di- and trichlorinated products. Although the Haring and Knol results refer to a gas-liquid reaction, they ignore the effects of mass transfer. According to their description of the way in which the green colouration of dissolved chlorine slowly spread throughout the liquid, it is almost certain that their product distribution was distorted by this macro-mixing with respect to non-uniformity of dissolved chlorine. In contrast a competitive/consecutive oxidation sequence with five reactions has shown how mass transfer of oxygen as well as vessel scale macro-mixing can be accommodated, even though micro-mixing effects were ignored (Mann et al., 1977).

The possibility that micro-mixing effects could distort product distributions in some complex and unforseen ways is raised by the anomolous behaviour shown in Figure 1 for a triplet sequence of $A+B \rightarrow R+B \rightarrow S+B \rightarrow T$. Under perfect mixing conditions, the intermediate products $R$ and $\mathrm{S}$ should appear sequentially in advance of the final product $\mathrm{T}$ as shown in Figure 1(a). However, it has been observed with respect to experimental brominations that the final product appears anomolously in advance of the two precursor 

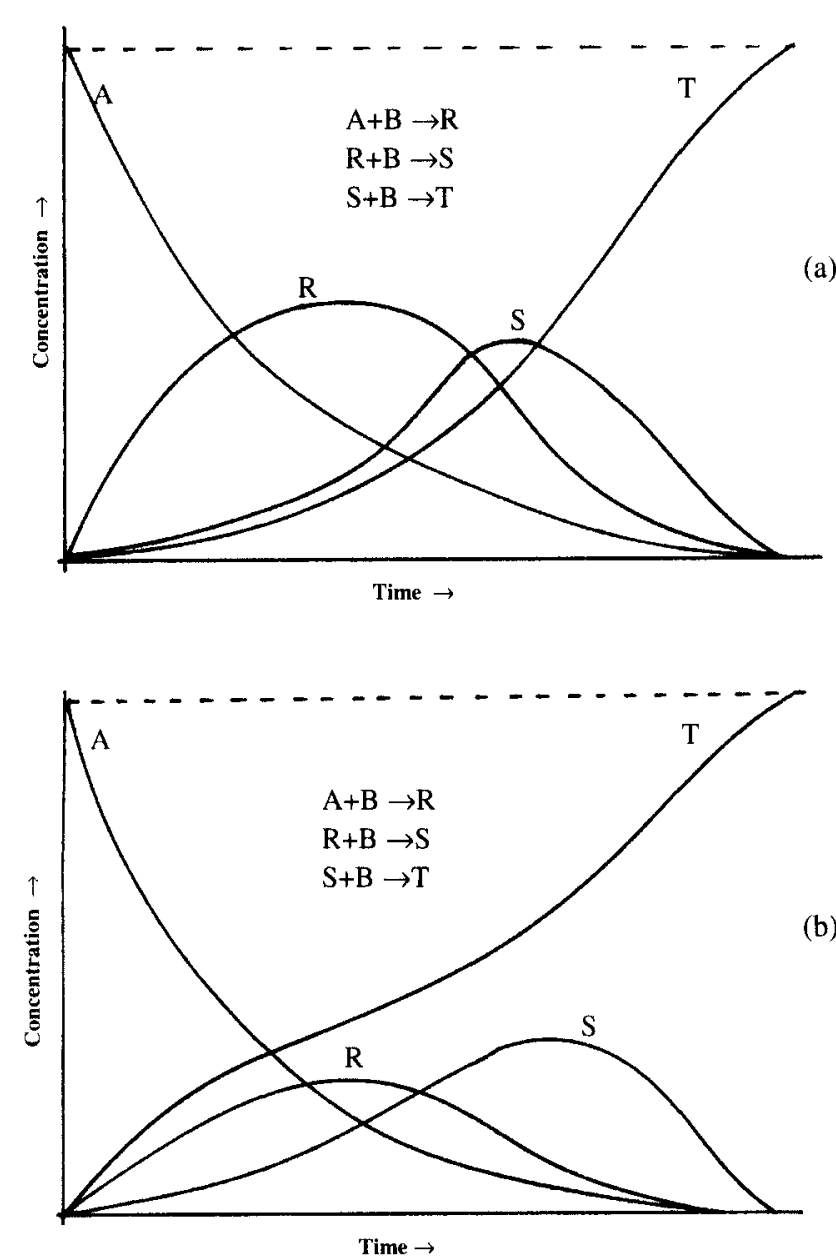

Figure 1 - Product distributions for a triplet competitive/consecutive reaction

a) product sequence under perfect mixing conditions,

b) experimentally observed anomalous behaviour.

intermediates, as in Figure 1(b). Whilst it is impossible for this anomolous behaviour to happen under perfect backmixing, under micro-mixing conditions it is possible for localised over-concentration of B to produce over-reaction locally to the final product. This anomaly poses new problems for the control of reactor product distribution and imposes a requirement for a more complete understanding of the role of both micro-mixing and macro-mixing on reactor behaviour.

Micro-mixing calculations for the pair of competitive/ consecutive reactions based upon a diffusion/reaction formulation have been thoroughly researched for the now classical diazotisation coupling of $\beta$-napthol (Bourne, 1983;) although the possible complexity of yield behaviour is restricted by the two-component stoichiometry, effectively giving only one degree of freedom for yield loss. By way of contrast, there is an additional degree of freedom for a triplet sequence of the form

$$
\begin{array}{lll}
\mathrm{A}+\mathrm{B} \rightarrow \mathrm{A}^{\prime} \mathrm{B} & & \mathrm{A}+\mathrm{B} \rightarrow \mathrm{R} \\
\mathrm{A}^{\prime} \mathrm{B}+\mathrm{B} \rightarrow \mathrm{A}^{\prime \prime} \mathrm{B}_{2} & \text { or } & \mathrm{R}+\mathrm{B} \rightarrow \mathrm{S} \\
\mathrm{A}^{\prime \prime} \mathrm{B}_{2}+\mathrm{B} \rightarrow \mathrm{A}^{\prime \prime \prime} \mathrm{B}_{3} & \text { or } & \mathrm{S}+\mathrm{B} \rightarrow \mathrm{T}
\end{array}
$$

giving rise to the three products A-mono-B, A-di-B and Atri-B. For many unsaturated hydrocarbons undergoing oxidation, hydrogenation and/or halogenation, the products can be additive, substitutive or both.
It is the purpose of the present paper to demonstrate an analysis of this triplet sequence of reactions under micromixing conditions using a simple non-deforming diffusionreaction formulation. In particular the question of the yield of products will be examined and the relative behaviour under perfect segregation and perfect mixing will be explored.

Difficulties relating to the interpretation of micro-mixing with stirred vessel fluid mechanics in typical reactor operation remains problematic. However there has been significant recent progress linking these in relation to copolymerisation in a stirred vessel (Atiqullah and Nauman, 1990) and the splitting of a feed stream to a stirred vessel in order to retain reactor productivity without sacrifice of the yield of an intermediate (Bourne and Hilber, 1990).

More recently, the concept of meso-mixing has been developed as a means of connecting micro-mixing at the molecular scale to macro-mixing at vessel scale (Baldyga et al., 1997). Two possible meso-mixing mechanics have been suggested involving either turbulent dispersion (Baldyga and Bourne, 1992) or inertial convective disintegration of large eddies (Baldyga et al., 1994).

In the analysis to be presented here, we focus solely on the impact of a simplified non-deformable micro-mixing framework for a set of three reactions, since this itself presents a significant degree of complexity. Extension of this analysis to integrate micro-mixing with reactor fluid mechanics will subsequently be necessary, so that the additional effects of micro-scale fluid mechanical deformations (important in the more advanced engulfment (EDD) formulation of Baldyga and Bourne, 1984), meso-mixing and macro-mixing can be determined. The sole effect of vessel scale macro-mixing on the distortion of batch reactor product distribution for a triplet set of reactions in a stirred vessel has already been explored (Mann and El-Hamouz, 1995). These two extremes of stagnant diffusion and reaction and locally perfect macro-mixing form asymptotes to behaviour between which the case of deforming micro-scale fluid mechanics will lie. This analysis therefore defines a useful practical boundary.

Most reaction engineering texts (for example Levenspiel, 1972) deal with the impact of the macro-scale extremes of plug and perfectly backmixed flow on chemical yield and selectivity for a triplet consecutive/competitive reaction but fail to consider the detailed impact of micro-mixing on more than just two reactions. However, the role of micro-mixing for a triplet set of parallel reactions taking place in a partially segregated environment has been reported (Bourne and $\mathrm{Yu}, 1994)$.

\section{Formulation of diffusion-reaction analysis}

Micro-mixing (mixing down to the molecular scale) is significant for relatively fast and instantaneous reactions whose half-life are similar to, or shorter than, the times over which equalisation of concentrations or homogenisation of mixtures of reagents can be expected to occur by a mixing process.

The mechanisms involved in the achievement of micromixing are envisaged as comprising engulfment, deformation and diffusion (so called EDD model, Baldyga and Bourne, 1984 and 1989). This EDD model is formulated as coupled non-linear parabolic partial differential equations of the general type

$$
\frac{\partial c_{i}}{\partial t}+u(x, t) \frac{\partial c_{i}}{\partial x}+D_{i} \frac{\partial^{2} c_{i}}{\partial x^{2}}+R_{i}(x, t) \ldots \ldots \ldots \ldots
$$


If the flow/deformation term is neglected, this equation is simplified and can be rendered dimensionless using

$$
C_{i}=c_{i} / c_{j o} ; \bar{X}=x / \delta_{o} ; T=D_{i} t / \delta_{o}^{2} \ldots \ldots \ldots \ldots \ldots
$$

so that

$$
\frac{\partial C_{i}}{\partial T}=\frac{\partial^{2} C_{i}}{\partial \bar{X}^{2}}+\frac{\delta_{o}^{2}}{D_{i} C_{j o}} R_{i} \ldots \ldots \ldots \ldots \ldots \ldots
$$

which assumes that $\delta_{o}$ remains constant (stretching of the laminae is ignored). The formulation of Equation (3) has been widely adopted, although Jenson (1983) applied spherical coordinates instead of this cartesian framework.

For the triplet sequence of competitive/consecutive reactions

$$
A+B \stackrel{k_{1}}{\longrightarrow} R+B \stackrel{k 2}{\longrightarrow} S+B \stackrel{k_{3}}{\longrightarrow} T
$$

the set of equations arising from Equation (3) are

$$
\begin{aligned}
\frac{\partial C_{A}}{\partial T} & =\frac{\partial^{2} C_{A}}{\partial \bar{X}^{2}}-\frac{\delta_{o}^{2}}{D_{A} C_{A o}}\left[k_{1} C_{A} C_{B}\right] \ldots \ldots \ldots \ldots \\
\frac{\partial C_{B}}{\partial T} & =\frac{\partial^{2} C_{B}}{\partial \bar{X}^{2}}-\frac{\delta_{o}^{2}}{D_{B} C_{A}}\left[k_{1} C_{A} C_{B}+k_{2} C_{B} C_{R}+k_{3} C_{B} C_{S}\right] \\
\ldots \ldots \ldots \ldots \ldots \ldots \ldots & \ldots \ldots \ldots \\
\frac{\partial C_{R}}{\partial T} & =\frac{\partial^{2} C_{R}}{\partial \bar{X}^{2}}+\frac{\delta_{o}^{2}}{D_{R} C_{A}}\left[k_{1} C_{A} C_{B}-k_{2} C_{B} C_{R}\right] \ldots \ldots \\
\frac{\partial C_{S}}{\partial T} & =\frac{\partial^{2} C_{s}}{\partial \bar{X}^{2}}+\frac{\delta_{o}^{2}}{D_{S} C_{A_{o}}}\left[k_{2} C_{B} C_{R}-k_{3} C_{B} C_{S}\right] \ldots \ldots \\
\frac{\partial C_{T}}{\partial T} & =\frac{\partial^{2} C_{T}}{\partial \bar{X}^{2}}+\frac{\delta_{o}^{2}}{D_{T} C_{A}}\left[k_{3} C_{B} C_{S}\right] \ldots \ldots
\end{aligned}
$$

This set of five parabolic pdes are solved subject to the boundary conditions

$$
\frac{\partial C_{i}}{\partial X}=0 \text { at } \bar{X}= \pm \frac{1}{2} \ldots \ldots \ldots \ldots \ldots \ldots \ldots
$$

The equations have been solved using a library package (NAG) available for the solution of partial differential equations. The algorithm D03PBF is based on the method of lines and restricted to one space variable. It is a general purpose code discretised in the space variable, thereby reducing any system of pdes to an initial value system of odes. Typically in this work, 8000 mesh points in space gave sufficient accuracy in timewise integration for the overall material balance, based upon integration for individual components, to be accurate to better than $1 \%$.

\section{Concentration profile predictions}

An exploration of the triplet reaction under micro-mixing has been undertaken using the parameter values listed in Table 1 .
TABle 1

Parameter Values for Triplet Consecutive/Competitive Reaction

\begin{tabular}{llc}
\hline \multicolumn{1}{c}{ Parameter } & Value & Units \\
\hline initial concentration of A & $C_{A o}=1.0$ & $\mathrm{~mol} \cdot \mathrm{m}^{-3}$ \\
initial concentration of B & $C_{B o}=3 C_{A o}$ & $\mathrm{~mol} \cdot \mathrm{m}^{-3}$ \\
diffusivity of A, R, S and T & $D_{A}=D_{R}=D_{S}=D_{T}$ & $\mathrm{~m}^{2} \cdot \mathrm{s}^{-1}$ \\
& $=1 \times 10^{-10}$ & \\
diffusivity of B & $D_{B}=5 D_{A}$ & $\mathrm{~m}^{2} \cdot \mathrm{s}^{-1}$ \\
reaction rate constants & $k_{1}=k_{2}=k_{3}=80$ & $\mathrm{~m}^{3} \cdot \mathrm{mol}^{-1} \cdot \mathrm{s}^{-1}$ \\
diffusion length & $\delta_{o}=50$ & $\mu \mathrm{m}^{2}$ \\
diffusion modulus for B & $M_{B}=k_{2} C_{B o} \delta_{o}^{2} / D_{B}$ & \\
& $=1200$ & \\
& & \\
\hline
\end{tabular}
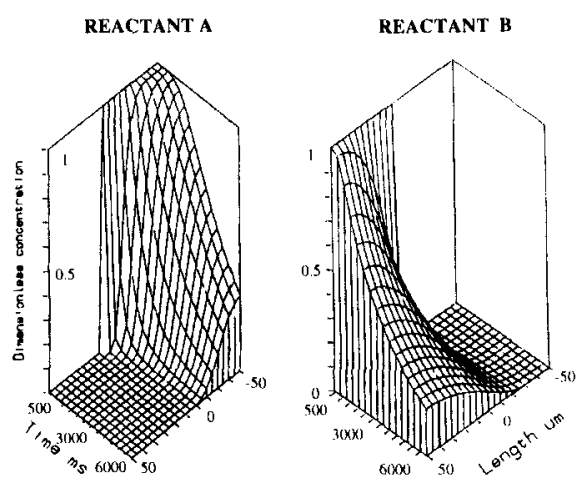

PRODUCT R

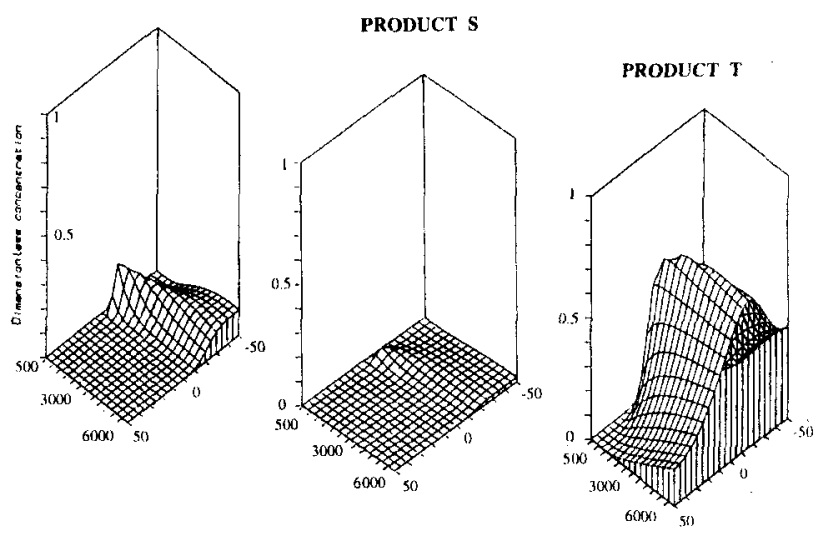

Figure 2 - Surface 3-D plots of species concentration profiles.

The use of $C B_{o}=3 C_{A O}$ is to provide for a stoichiometric equivalence of $\mathrm{A}$ and $\mathrm{B}$ sufficient to give completion of the reaction to the final product $\mathrm{T}$, though this is likely to be prejudicial to the yield of the intermediates $\mathrm{R}$ and $\mathrm{S}$. In line with the probable utility of this set of three reactions for oxidations, halogenations and hydrogenations, the component $B$ is allocated a five times higher diffusivity and the diffusivities of the primary reagent $A$ and three products are taken to be equal. A diffusional length of $50 \mu \mathrm{m}$ has been chosen, reflecting the fact that in typical stirred vessels $\delta_{o}$ is expected to vary between 10 and $100 \mu \mathrm{m}$ (Villermaux, 1983), so this represents a suitably intermediate value. The diffusion modulus $M_{B}$ takes a value of 1200 , placing the reactions firmly in a micro-mixing influenced regime.

The evolution of each of the species can be very compactly represented using 3-D surface plots as shown in Figure 2. This figure shows that the first product $\mathrm{R}$ is formed within the A rich zone. Initially at short times less than about $3 \mathrm{~s}, \mathrm{R}$ forms a profile showing a distinct maximum. This arises from the initial high local rate of formation of $R$ 


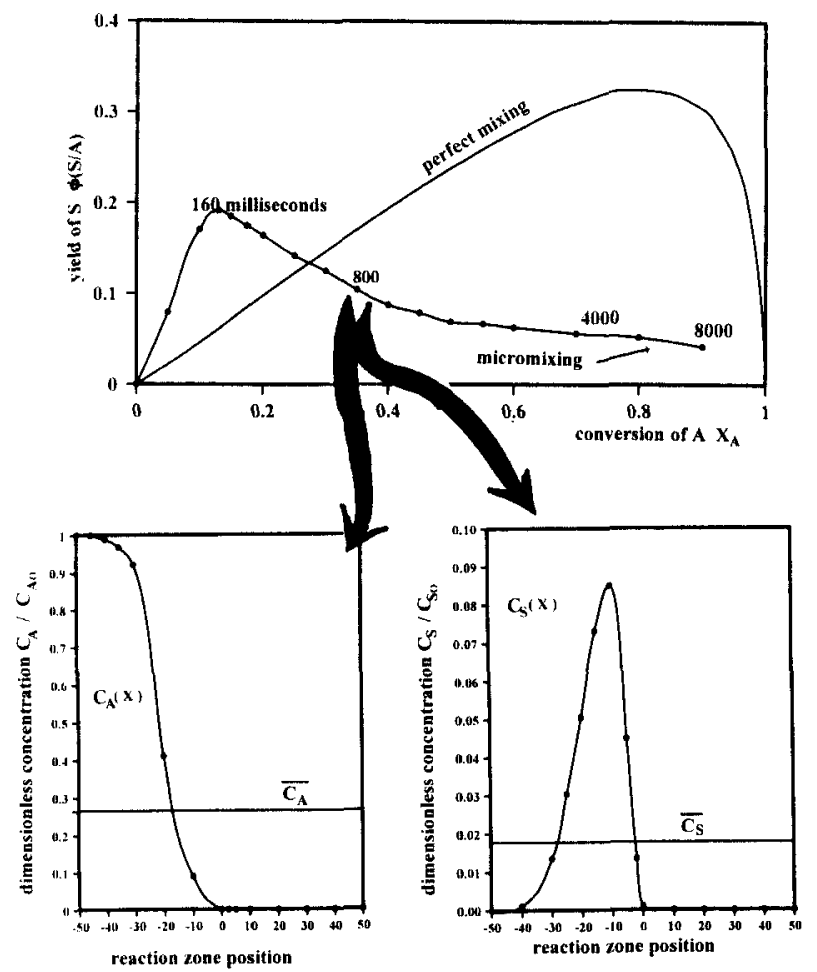

Figure 3 - Yield of $\mathrm{S}$ vs $X_{A}$ : comparison of perfect mixing and micromixing.

as $\mathrm{A}$ and $\mathrm{B}$ interdiffuse at relatively high concentrations. Thereafter, as the concentrations of reagents decline, the profile of R spreads out and the "peak" in its concentration disappears by self-diffusion. The central product $\mathrm{S}$ appears at much lower concentration levels with a scarcely discernible peak value. In contrast, the final product $\mathrm{T}$ takes some time to develop a pronounced peak value, but this persists for longer times and is less ameliorated by its self-diffusion tendency. Figure 2 shows that the "peaks" of R, S and $T$ are all confined to the A-rich "side" of the interface between $A$ and $B$. The simulations show that the spatial distribution of the final product $\mathrm{T}$ shows a distinct non-uniformity despite the apparent opportunity for production by diffuse reaction and equalisation by self-diffusion. On the other hand, the product $S$ appears in relatively low concentration being sparingly formed from A-B reaction and consumed by the following reaction with $B$.

However, in chemical reaction engineering terms, the yield of individual products is more important than any specific details of their concentration profiles.

\section{Yield behaviour of the middle product $S$}

Following the terminology adopted by Levenspiel (1972) based on the earlier work of Denbigh (1966), the local instantaneous yield of $\mathrm{S}$ with respect to reagent $\mathrm{A}$ is given by

$$
\begin{aligned}
& \psi_{x}\left[\frac{\mathrm{S}}{\mathrm{A}}\right]=\frac{\text { rate at which product } S \text { is produced }}{\text { rate at which reagent } \mathrm{A} \text { is consumed }} \\
& =\frac{k_{2} C_{R} C_{B}-k_{3} C_{S} C_{B}}{k_{1} C_{A} C_{B}} \\
& \psi_{x}\left[\frac{\mathrm{S}}{\mathrm{A}}\right]=\frac{k_{2} C_{R}(x)-k_{3} C_{S}(x)}{k_{1} C_{A}(x)} \ldots \ldots \ldots
\end{aligned}
$$

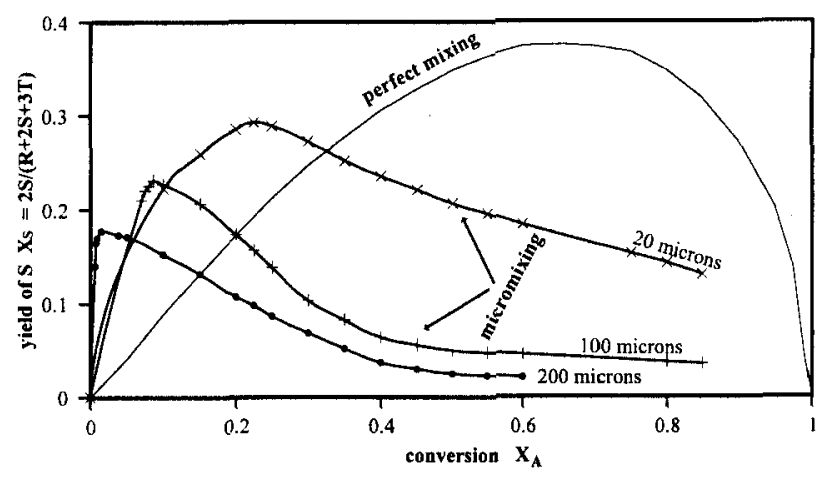

Figure $4-$ Yield of $\mathrm{S}$ vs $X_{A}$ : Effect of micromixing zone thickness.

This instantaneous yield is particular to the local planar position $x$ within $-\delta_{o}<x<\delta_{\theta}$. The overall instantaneous yield at a particular instant of time is then given by

$$
\Phi_{t}\left[\frac{\mathrm{S}}{\mathrm{A}}\right]=\frac{\text { mols of S produced }}{\text { mols of A consumed }}=\frac{\int_{-\delta_{o}}^{+\delta_{o}} C_{S}(x) d x}{1-\frac{1}{2 \delta_{o}} \int_{-\delta_{o}}^{+\delta_{o}} C_{A}(x) d x}
$$

The overall yield is also expressible in terms of the mixing-cup concentration which would result if the A-rich and B-rich slabs were to be instantaneously perfectly mixed. This mixing cup concentration for a component $\bar{C}_{I}$ is denoted by and given by

$$
\bar{C}_{I}=\frac{1}{2 \delta_{o}} \int_{-\delta_{o}}^{+\delta_{o}} C_{I}(x) d x \ldots \ldots \ldots \ldots \ldots \ldots
$$

so that the overall yield after a micro-mixing time $t$ would also be given by

$$
\Phi_{t}\left[\frac{\mathrm{S}}{\mathrm{A}}\right]=\frac{\bar{C}_{S}}{1-\bar{C}_{A}}=\frac{\bar{C}_{S}}{\bar{X}_{A}} \ldots \ldots \ldots \ldots \ldots
$$

Figure 3 then shows the calculated values of $\Phi[S / A]$ versus the overall conversion of $\mathrm{A}\left(\bar{X}_{A}\right)$, for the parameter values of Table 1. It is clear from Figure 3 that the diffusion and reaction processes of micro-mixing initially give a much superior yield relative to that which would be produced by perfect mixing. The yields from the two mixing extremes are equal at $\bar{X}_{A}=0.30$. Thus at lower overall conversions, micro-mixing improves the yield of $S$ with respect to $A$. At higher conversions, perfect mixing would give superior yields. With micro-mixing a maximum yield of S of $19 \%$ is achieved at $\bar{X}_{A}=13 \%$, whereas at this conversion perfect mixing would give a yield of only some $6 \%$. Figure 3 also shows the micro-mixing times which correspond to the levels of overall conversion achieved. At $800 \mathrm{~ms}$, the two concentration profiles for $C_{A}(x)$ and $C_{S}(x)$ are shown side by side. It is these concentration profiles that give the overall yield after $800 \mathrm{~ms}$ according to Equation (11). The mixing cup values $\bar{C}_{s}$ and $\bar{C}_{A}$ are also shown for comparison purposes.

Figure 4 indicates how this overall yield of $S$ is affected by the initial thickness of the A-rich and B-rich adjacent 'slabs' of fluid. For the thicker $200 \mu \mathrm{m}$ case, the yield of $\mathrm{S}$ 

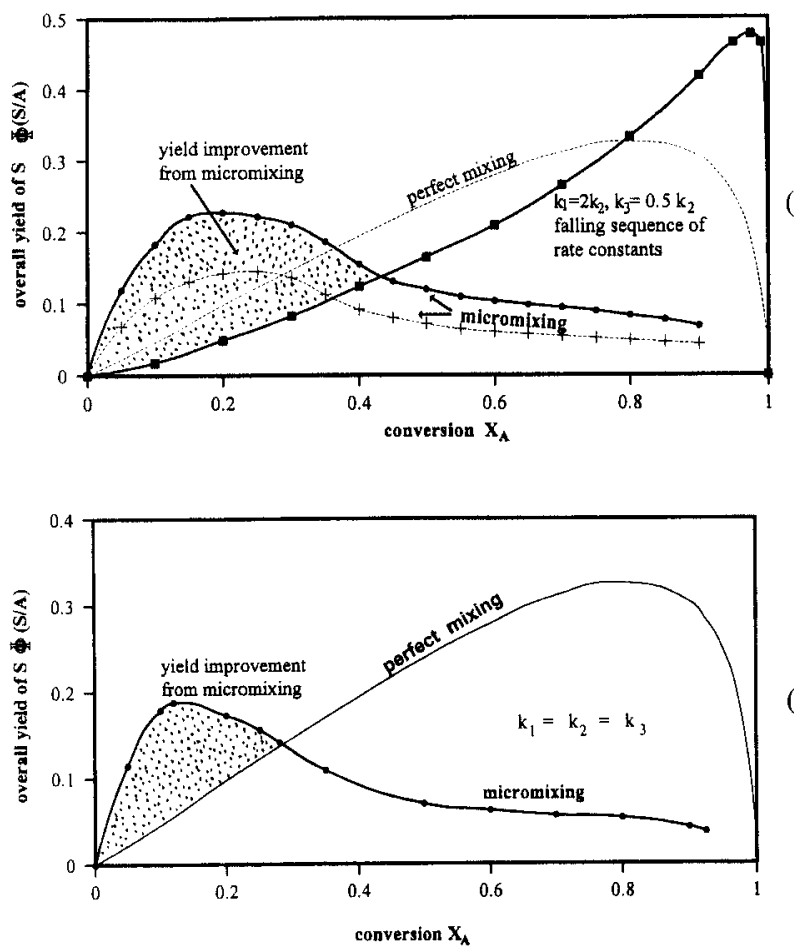

(b)

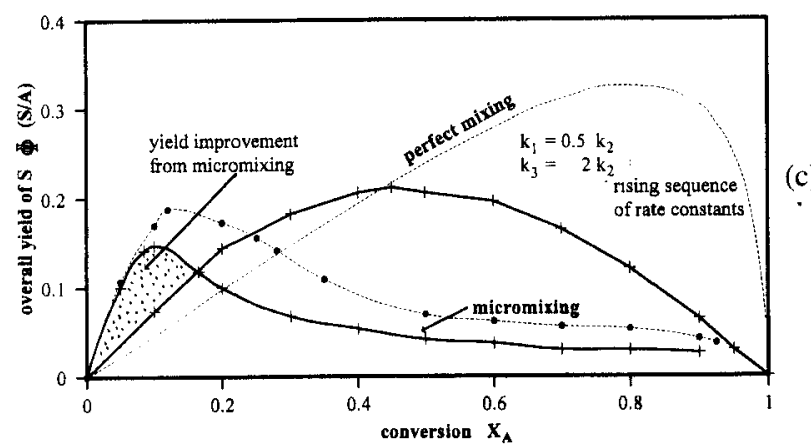

Figure 5-Effect of falling and rising sequences of rate constants on $\Phi_{t}$ (S/A)

(a) falling sequence of rate constants,

(b) equal rate constants,

(c) rising sequence of rate constants.

is improved at low conversions less than about $5 \%$. For the much thinner $20 \mu \mathrm{m}$ case, at less than $15 \%$ conversion the yield of $S$ is smaller, but reaches a higher maximum of $\approx 23 \%$ at a conversion of about the same value. Figure 4 suggests the intriguing possibility of optimising yield, along the locii of maxima, by using a controlled reduction in $\delta_{o}$ (from some initial relatively high value). Some form of engineered stretching is implied, as overall conversion advances with increasing time.

Another measure of the yield of products in this triple reaction case is with respect to the second reagent $B$. Adopting the symbolism used by Bourne (1983), $X_{S}$ is the yield of $S$ denoted by

$$
X_{S}=\frac{\text { moles of B incorporated in product } S}{\text { total moles of B consumed }}=\frac{2 S}{3 \mathrm{~T}+2 \mathrm{~S}+\mathrm{R}}
$$

This gives rise to yield values which are somewhat different from those based on A although similar in pattern.

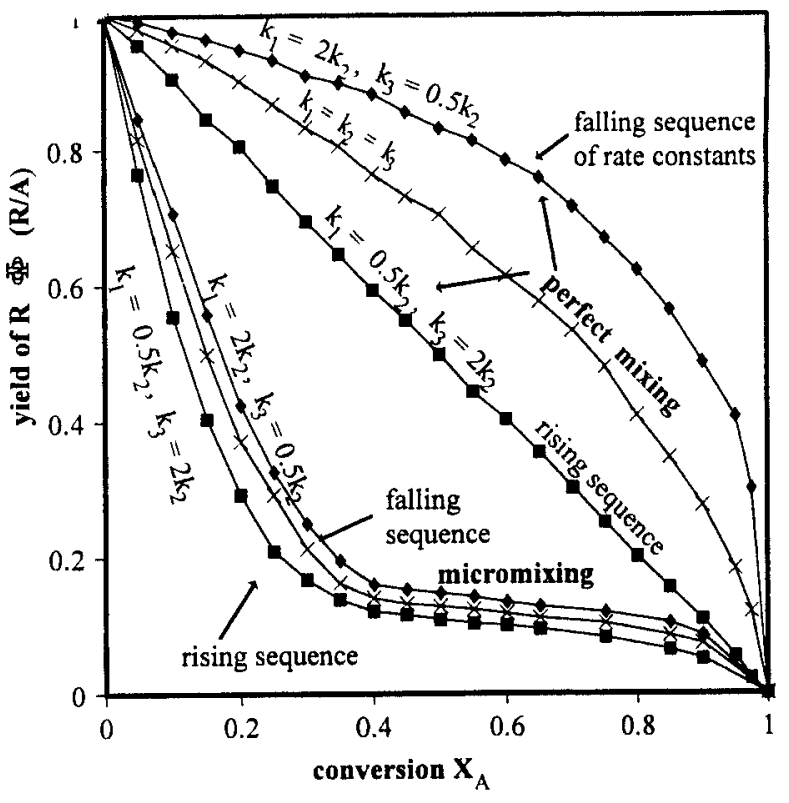

Figure $6-$ Yield of $\mathrm{R}-\Phi_{t}(\mathrm{R} / \mathrm{A})$.

The relative importance of yields based on A or B are dependent upon the relative costs of the reagents. Usually the yield based on the most expensive reagent would be the most significant.

Finally, Figure 5 shows the impact of the relative sizes of the three rate constants. Previous figures referred to $k_{1}=k_{2}$ $=k_{3}$ and Figure 5(a) indicates the effect when the rate is higher for $\mathrm{R}$ and lower for $\mathrm{T}$ (referred to here as a falling sequence of rate constants) using $k_{1}=2 k_{2}$ and $k_{3}=1 / 2 k_{2}$ Thus in Figures 5(a) and (c) the results pivot about the middle rate constant $\mathrm{k}_{2}$. From Figure 5 it is evident that micro-mixing gives an enhanced yield relative to perfect mixing for the falling sequence of rate constants. Hence with a rising sequence, for which the formation of $\mathrm{T}$ is relatively faster, the scope for improved yield of $S$, using micro-mixing (complete segregation) in preference to perfect mixing, is very much less.

\section{Yield behaviour of initial and final products ' $R$ ' and ' $T$ '}

So far, this analysis of the triplet sequence of reactions has concentrated on the central product $S$. For completeness, the yield results for $\mathrm{R}$ and $\mathrm{T}$ will be briefly presented.

Figure 6 shows a comparison between micro-mixing and perfect mixing for the case of equal rate constants as well as the rising and falling sequences. It can be seen that micromixing always reduces the yield of the first product. (This is well known, since consideration of $\mathrm{R}$ by itself amounts to the well researched doublet reaction case). The yield of $R$ with respect to $A, \Phi_{t}[R / A]$ is always high at low conversion of $\mathrm{A}$. The impact of the rising/falling sequencing is more pronounced for perfect mixing. At middle range conversions, the yield loss caused by micro-mixing is dramatic. At around $50 \%$ conversion of $\mathrm{A}$, micro-mixing reduces the potential yield from $85 \%$ to below $15 \%$.

Figure 7 then shows results for $\Phi_{t}[\mathrm{~T} / \mathrm{A}]$. The yield of the final product $T$ is greatly improved by the restricted mixing implied by perfect segregation, where reaction can only proceed by mutual interdiffusion. For equal rate constants at the $50 \%$ conversion level, perfect mixing would give only $1 \%$ yield of $\mathrm{T}$, which can be increased to about $80 \%$ by permitting 


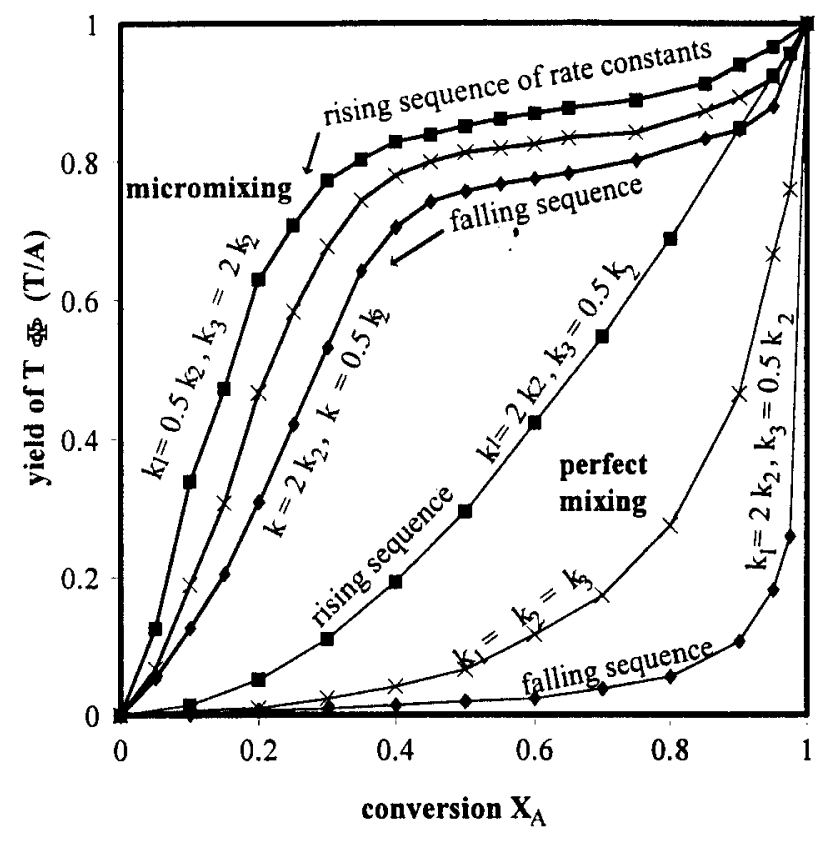

Figure $7-$ Yield of $T-\Phi_{t}$ (T/A).

only diffusional mixing. The relative yield improvement with micro-mixing is most marked for the falling sequence of rate constants.

\section{Optimal combinations of mixing}

Figure 8 presents some initial results on maximising the yield of the central intermediate ' $S$ ' by using micro-mixing followed by perfect mixing, so as to improve the available yield at a given conversion.

For equal rate constants (as in Table 1), Figure 8 shows the predicted behaviour if micro-mixing is followed by perfect mixing. If perfect mixing is initiated at the peak value $\Phi_{t}$ [S/A], there is a large drop in the selectivity relative to the continuation of micro-mixing. This then gives rise to only a marginal improvement in the yield of $\mathbf{S}$ over the conversion range 30 to $40 \%$ which amounts to about $2 \%$ (though this is itself a $10 \%$ relative improvement). However, beyond $40 \%$ conversion, the contribution of micro-mixing and perfect mixing is inferior to the sole use of perfect mixing. According to Figure 8, between $10 \%$ and $30 \%$ conversion of $\mathrm{A}$, the yield of $\mathrm{S}$ is best maximised by using only micromixing.

\section{Conclusions}

1. A simplified non-deforming micro-mixing diffusion and reaction formulation for the triplet reaction sequence

$$
\mathrm{A}+\mathrm{B} \stackrel{k_{1}}{\mathrm{R}}+\mathrm{B} \frac{k_{2}}{2} \mathrm{~S}+\mathrm{B} \stackrel{k_{3}}{\mathrm{~T}} \mathrm{~T}
$$

when posed as a set of parabolic differential equations, may be readily solved by an all-purpose library integration (NAG) sub-routine.

2. Using numerical values considered to be relevant to typical substitutive and/or additive halogenation reactions it has been shown that: $i)$ the primary product $R$ is always favoured by perfect mixing, ii) the central intermediate $S$

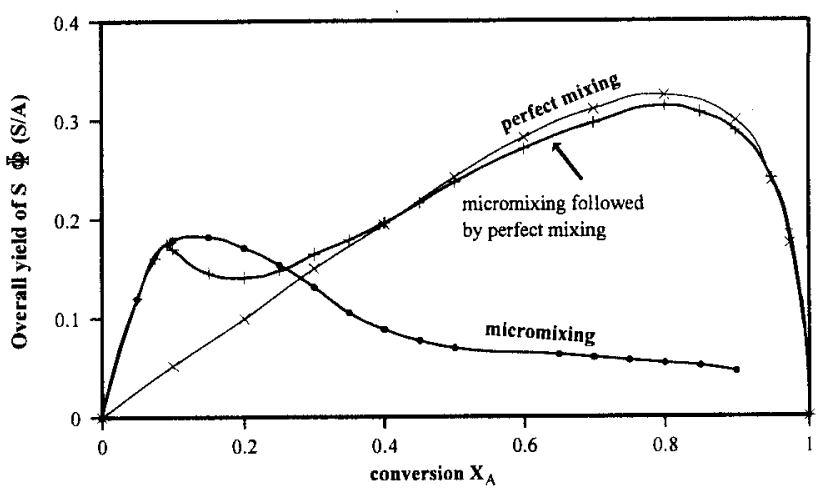

Figure $8-$ Yield of $S-\Phi_{t}(\mathrm{~S} / \mathrm{A})-$ Effect of combinations of mixing.

shows improved yields with respect to both reagents $A$ and $B$ under perfect initial segregation conditions whereby reaction proceeds by micro-mixing, iii) the ultimate product $\mathrm{T}$ is always favoured by micro-mixing.

3. The predicted effect of different lamella thicknesses upon the yield of the central intermediate $S$, suggests that the yield of S may be able to be improved using a reactor which utilises an engineered stretching of lamellae as reaction proceeds.

4. At intermediate conversion levels, the yield of S may be maximised by using micro-mixing followed by perfect mixing.

5. This simplified non-deforming analysis of micro-mixing forms an asymptotic limit for yield and selectivity behaviour for a triplet competitive-consecutive reaction. The analysis needs extending to incorporate the physically more realistic effects of deformation and engulfment. Experimental work is also required to check whether prior findings of the irrelevance of species diffusivities for simpler kinetics (Bolzern et al., 1985; Baldyga and Henczka, 1997) will apply to this larger set of reactions exhibiting more severely non-linear reaction kinetics.

\section{Nomenclature}

$c_{i}=$ concentration of component $\mathrm{i}=\mathrm{A}, \mathrm{B}, \mathrm{R}, \mathrm{S}$ and $\mathrm{T}$ $\left(\mathrm{kmol} \cdot \mathrm{m}^{-3}\right)$

$C_{i} \quad=$ dimensionless concentration of component $\mathrm{i}$ with respect to $C_{A O}$

$\bar{C}_{i} \quad=$ mixing cup concentration of component $\mathrm{I}\left(\mathrm{kmol} \cdot \mathrm{m}^{-3}\right)$

$D_{i} \quad=$ diffusivity of component $\mathrm{i}\left(\mathrm{m}^{2} \mathrm{~s}^{-1}\right)$

$k_{n}=$ nth reaction rate constant $(\mathrm{n}=1,2,3)\left(\mathrm{m}^{3} \cdot \mathrm{mol}^{-1} \cdot \mathrm{s}^{-1}\right)$

$R_{i} \quad \quad=$ reaction rate for ith component $\left(\mathrm{kmol} \cdot \mathrm{m}^{-3} \cdot \mathrm{s}^{-1}\right)$

$T \quad=$ dimensionless time

$t \quad=$ time (s)

$u \quad=$ velocity $\left(\mathrm{m} \cdot \mathrm{s}^{-1}\right)$

$\bar{X} \quad=$ dimensionless position

$\bar{X}_{A} \quad=$ overall average conversion of $\mathrm{A}$

$X_{S} \quad=$ yield of product $\mathrm{S}$ with respect to reagent $\mathrm{B}$ $\left(X_{s}=2 \mathrm{~S} /(\mathrm{R}+2 \mathrm{~S}+3 \mathrm{~T})\right)$

$x \quad=$ position (one dimensional) $(\mathrm{m})$

\section{Greek letters}

$\delta_{0}=$ thickness of fluid lamella $(\mu \mathrm{m})$

$\Phi_{t}(\mathrm{I} / \mathrm{J})=$ overall yield of product I with respect to reagent $\mathrm{J}$ after time (t)

$\Psi_{x}(\mathrm{I} / \mathrm{J})=$ instantaneous local yield of product I with respect to reagent $(\mathrm{J})$ 


\section{References}

Atiquallah, M. and E. B. Nauman, "A Model and Measurement Technique for Micro-mixing in Polymerisation Reactors", Chem. Eng. Sci. 45, 1267-1279 (1990).

Baldyga, J., J. R. Bourne and S. J. Hearn, "Interaction between Chemical Reactions and Mixing on Various Scales", Chem. Eng. Sci. 52, 457-466 (1997).

Baldyga, J., J. R. Bourne and B. Zimmerman, "Investigation of Mixing in a Jet Reactor using fast Competitive - Consecutive Reactions", Chem. Eng. Sci. 49, 1937-1946 (1994).

Baldyga, J. and J. R. Bourne, "A Fluid Mechanical Approach to Turbulent Mixing and Chemical Reaction: Part I, Inadequacies of Available Methods", Chem. Eng. Comm. 28, 231-281 (1984).

Baldyga, J. and J. R. Bourne, "Interactions between Mixing on Various Scales in Stirred Tank Reactor", Chem. Eng. Sci. 47, 1837-1848 (1992).

Baldyga, J. and J. R. Bourne, "Simplification of Micro-mixing Calculations. I Derivation and Application of New Model", Chem. Eng. J. 42, 83-92 (1989).

Baldyga, J. and M. Henczka, "Turbulent Mixing and Parallel Chemical Reactions in a Pipe: Application of a Closure Model", Réc. Prog. en Génie des Procédés (9th European Conference on Mixing, Paris), 11, 341-349 (1997).

Bolzern, O., J. R. Bourne and G. Tovstiga "Micromixing and Fast Reaction in the Core of Turbulent Pipe Flow", Chem. Eng. Comm., 38, 1-8 (1985).

Bourne, J. R., "Mixing on the Molecular Scale (Micro-mixing)", Chem. Eng. Sci. 38, 5-8 (1983).

Bourne, J. R. and C. P. Hilber, "The Productivity of Micro-mixingControlled Reactions: Effect of Feed Distribution in Stirred Tanks", Trans. I.Chem.Eng. 68(A), 51-56 (1990).

Bourne, J. R. and S.Yu, "Investigation of Micromixing in Stirred Tank Reactors using Parallel Reactions", Ind. Eng. Chem. Res. 33, 41-55 (1994).

Danckwerts, P. V., "The Effect of Incomplete Mixing on Homogeneous Reaction", Chem. Eng. Sci. 8, 93-102 (1958).

Denbigh, K. G., "Chemical Reactor Theory: An Introduction", Cambridge University Press, UK (1966).

Haring, H. G., and H. W. Knol, "Photochemical Side-Chain Chlorination of Toluene", Chem. Proc. Eng. 45, 560-567 (1964).

Jenson, V. G., "A Model for Mixing with Fast Chemical Reactions", Chem. Eng. Sci. 38, 1151-1157 (1983).

Levenspiel, O., "Chemical Reaction Engineering", 2nd Edn., Wiley, New York, NY (1972).
MacMullin, R. B., "Distribution of Reaction Products in Benzene Chlorination: Batch vs Continuous Process Procedures", Chem. Eng. Prog. 44, 183-190 (1948).

Mann, R. and A. M. El-Hamouz, "A Product Distribution Paradox on Scaling Up a Stirred Batch Reactor", AIChE J. 41, 855-867 (1995).

Mann, R., J. C. Middleton and I. B. Parker, "Deteriorating Mixing Quality and Yield Losses on Scaling - Up Gas Liquid Reactors", Proc. 2nd Europ. Conf. Mixing, Cambridge (BHRA) UK, F3 (1977).

Mehta, R. V. and J. M. Tarbell, "Four Environment Model of Mixing and Chemical Reaction: Part 1", AIChE J. 29, 320-329 (1983).

NAG (Numerical Algorithms Group), Fortran Library Manual Mark 13, Vol. 3, DO3PBF (1988).

Ritchie, B. W. and A. H. Tobgy, "A Three Environment Mixing Model for Chemical Reactors with Arbitrary Separate Feed Streams", Chem. Eng. J. 17, 173-182 (1979).

Russel, T. W. F. and D. T. Buzzelli, "Reactor Analysis and Process Synthesis for a Class of Complex Reactions", Ind. Eng. Chem. Proc. Des. Dev. 8, 2-9 (1969).

Spielman, L. A. and O. Levenspiel, "A Monte Carlo Treatment for Reacting and Coalescing Dispersed Phase Systems", Chem. Eng. Sci. 20, 247-254 (1965).

Treleaven, C. R. and A. H. Togby, "Monte Carlo Method of Simulating Micro-mixing in Chemical Reactors", Chem. Eng. Sci. 27, 1497-1513 (1972).

Villermaux, J., "Mixing in Chemical Reactors", ACS Symposium Series, 226, 135-155 (1983).

Villermaux, J. and R. David, "Prediction of the Yield of a Consecutive/Competitive Reaction in a CSTR with Separate Feed Streams by a Macro/Micro Mixing Model", Paper 115d, AIChE Annual Meeting, San Francisco, (1989).

Weinstein, H. and R. V. Adler, "Micro-mixing Effects in Continuous Chemical Reactors", Chem. Eng. Sci. 22, 65-75 (1967).

Zweitering, T. N., "The Degree of Mixing in Continuous Flow Systems", Chem. Eng. Sci. 11, 1-15 (1959).

\footnotetext{
Manuscript received February 23, 1998; revised manuscript received June 4, 1998; accepted for publication June 5, 1998.
} 